\title{
On the Use of Emoticons in Open Source Software Development
}

\author{
Maëlick Claes \\ M3S, ITEE, University of Oulu, \\ Finland \\ maelick.claes@oulu.fi
}

\author{
Mika Mäntylä \\ M3S, ITEE, University of Oulu, \\ Finland \\ mika.mantyla@oulu.fi
}

\author{
Umar Farooq \\ M3S, ITEE, University of Oulu, \\ Finland \\ umar.farooq@oulu.fi
}

\begin{abstract}
Background: Using sentiment analysis to study software developers' behavior comes with challenges such as the presence of a large amount of technical discussion unlikely to express any positive or negative sentiment. However, emoticons provide information about developer sentiments that can easily be extracted from software repositories. Aim: We investigate how software developers use emoticons differently in issue trackers in order to better understand the differences between developers and determine to which extent emoticons can be used as in place of sentiment analysis. Method: We extract emoticons from 1.3M comments from Apache's issue tracker and 4.5M from Mozilla's issue tracker using regular expressions built from a list of emoticons used by SentiStrength and Wikipedia. We check for statistical differences using Mann-Whitney U tests and determine the effect size with Cliff's $\delta$. Results: Overall Mozilla developers rely more on emoticons than Apache developers. While the overall ratio of comments with emoticons is of $2 \%$ and $3.6 \%$ for Apache and Mozilla, some individual developers can have a ratio above $20 \%$. Looking specifically at Mozilla developers, we find that western developers use significantly more emoticons (with large size effect) than eastern developers. While the majority of emoticons are used to express joy, we find that Mozilla developers use emoticons more frequently to express sadness and surprise than Apache developers. Finally, we find that developers use overall more emoticons during weekends than during weekdays, with the share of sad and surprised emoticons increasing during weekends. Conclusions: While emoticons are primarily used to express joy, the more occasional use of sad and surprised emoticons can potentially be utilized to detect frustration in place of sentiment analysis among developers using emoticons frequently enough.
\end{abstract}

\section{CCS CONCEPTS}

- General and reference $\rightarrow$ Empirical studies; • Information systems $\rightarrow$ Sentiment analysis; • Human-centered computing $\rightarrow$ Open source software; $\bullet$ Computing methodologies $\rightarrow$ Natural language processing;

\section{KEYWORDS}

Emoticons, Sentiment Analysis, Repository Mining, Emoticon Usage, Emotions, Open Source Software Development

ESEM '18, October 11-12, 2018, Oulu, Finland

(c) 2018 Copyright held by the owner/author(s). Publication rights licensed to the Association for Computing Machinery.

This is the author's version of the work. It is posted here for your personal use. Not for redistribution. The definitive Version of Record was published in ACM / IEEE International Symposium on Empirical Software Engineering and Measurement (ESEM) (ESEM '18), October 11-12, 2018, Oulu, Finland, https://doi.org/10.1145/3239235.3267434.
ACM Reference Format:

Maëlick Claes, Mika Mäntylä, and Umar Farooq. 2018. On the Use of Emoticons in Open Source Software Development. In ACM / IEEE International Symposium on Empirical Software Engineering and Measurement (ESEM) (ESEM '18), October 11-12, 2018, Oulu, Finland. ACM, New York, NY, USA, 4 pages. https://doi.org/10.1145/3239235.3267434

\section{INTRODUCTION}

Decentralized online software development has grown during the past decades to a point where thousands of developers collaborate on a daily basis using electronic communication means. In software engineering, many empirical studies have studied developers interactions and behavior with mining repository techniques.

In particular, sentiment analysis has become more and more popular in the past years to study developers' behavior at a large scale. Because most gold standards for sentiment analysis are not based on software engineering data [1], generic sentiment analysis tools can often perform poorly [2] for analyzing software development data. Thus, software engineering specific gold standards for sentiment analysis have been developed [3, 4]. Although such approaches improve sentiment analysis tool performance it may not be enough [5]. Additionally, they require tedious manual labeling. Emoticon usage on large software engineering text masses may lead to automated sentiment labeling and dictionary building [6].

One of the challenge with sentiment analysis in a software engineering context is the large amount of neutral sentences due to the technical nature of discussion. Moreover, it can also be difficult for human beings [7] to interpret emotions based solely on text. Emoticons are a popular way to add information about expressed emotion in online textual conversations and can be used to improve sentiment analysis tools [8]. However, emoticon usage can vary between persons, culture or from one context to another [9].

In this paper, we present the early results of an investigation of the differences in usage of emoticons in open source projects from Mozilla and Apache. Our goal is to understand how developers use emoticons differently and to which extent emoticons can be used as a proxy to measure developer behaviors. We define the following research questions:

- How does the use of emoticons vary among developers?

- What kind of emotions are expressed with emoticons?

- How does the use of emoticons vary across a week?

The remaining of this paper is structured as follows. In section 2 we present the related work. In section 3 we present the methodology used to collect data, and how we extracted emoticons from issue comments. In section 4 we present preliminary results for each of our research questions. In section 5, we present the limits of our study and we finally conclude in section 6 . 


\section{RELATED WORK}

In the last two decades, emoticons have been widely used for communication on the Internet and considered as the paralanguage of the Web [10]. As identified in [11], 6.1\% of electronic mails contains emoticons and $13.2 \%$ of the posts on UseNet newsgroup have emoticons [12]. Emoticons are exploited in both lexicon based and machine learning approaches of sentiment analysis to improve the accuracy of existing lexicon dictionary based sentiment analysis [13-18]. As shown in [19] the prediction capability of tweets as positive and negative is considerably enhanced when the emoticons are considered while training the classifier. One of the issue with traditional machine learning technique for sentiment classification is the dependency on topic, domain or even on natural language. An effort is made in [20] in order to reduce this dependency by training the classifier using an emoticon corpus.

In most literature, emoticons are investigated in social media contents. Differences in emoticon usage have been found between gender, showing that in a same sex context, females use more emoticons than males, while in a mixed sex context, male tend to adopt the same standard of expression [21]. Park et al. [9] showed that emoticon usage differs between geographical area and social network. Moreover, they are generally positive and strongly negative sentences generally don't contain emoticons. Miller et al. [22] pointed out that there can be disagreement among users about emojis being positive, negative or neutral.

However, in this study, we are investigating it in more formal and technical communication of developers during software development. According to the best of our knowledge, this is the first effort to study the emoticons in software engineering in order to primarily focus on how developers use emoticons and which types of emoticons are more frequent.

\section{METHODOLOGY}

In this section, we present first the methodology used for extracting data from Apache's and Mozilla's issue trackers. Then we give details on how we identified emoticons from the data alongside some preliminary numbers about the presence of emoticons in Mozilla and Apache comments.

\subsection{Data extraction}

To answer our research questions, we mined data from Mozilla's ${ }^{1}$ and Apache's ${ }^{2}$ issue trackers. We specifically extracted the history of all comments with associated authors, projects and timestamps using Perceval from the GrimoireLab tool chain [23]. Because issue tracker comments don't contain the timezone of the commenter, we also extracted information with Perceval about commits from Mozilla's Mercurial repositories ${ }^{3}$ and Apache's Git repositories ${ }^{4}$. In order to link developer timezones from the commit repositories to their isssue tracker comments, we performed a basic merging of the different authors' identities. We grouped together identities using the same name or email addresses after cleaning. Two of the authors manually checked the result in order to avoid any false

\footnotetext{
${ }^{1}$ https://bugzilla.mozilla.org

${ }^{2}$ https://issues.apache.org/jira/

${ }^{3}$ https://hg.mozilla.org

${ }^{4}$ https://git.apache.org/
}

positive. We only kept the (merged) developers who had made at least 100 commits. We end up with 102 Apache projects and 66 Mozilla projects with at least 1000 comments. In total, these amounts to $1.3 \mathrm{M}$ Apache comments and 4.5M Mozilla comments.

\subsection{Identifying emoticons}

In order to detect emoticons, we first used NLoN [24] to discard lines of text that are not natural language, such as code and execution traces. We used the list of ASCII emoticons used by SentiStrength and completed it with common ASCII emoticons from Wikipedia $^{5}$. From this list, we built regular expressions in order to extract the emoticons present in comments. We found 84 of the 107 emoticons from our list that were used at least once in a comment. We manually classified the emoticons based on basic emotions defined by Plutchik [25]: joy (42 emoticons), sadness (11), surprise (25), anger (4) and unknown (2). We made our list of emoticons and associated emotions available at https://github.com/M3SOulu/ ESEM2018-Emoticons-Emotions-List.

Table 1: Number of authors, comments and emoticons, percentage of comments with at least one emoticon, and percentage of authors having used at least one emoticon for Apache and Mozilla.

\begin{tabular}{l|r|r|r} 
source & comments & authors & emoticons \\
\hline Apache & $1,262,833(2.12 \%)$ & $956(68.83 \%)$ & 27,903 \\
Mozilla & $4,466,817(3.65 \%)$ & $472(97.46 \%)$ & 172,868
\end{tabular}

Table 1 shows for Apache and Mozilla the number of unique developers found in the issue trackers and the percentage who has used at least one emoticon, the total number of comments and percentage of comments with at least one emoticon, and the total number of emoticons detected. Overall most comments only contain at most one emoticon. Only 3.7\% of Apache comments with emoticons and $4.9 \%$ of Mozilla comments with emoticons contain 2 emoticons. Less than $1 \%$ of the comments with emoticons contain more than 2 emoticons.

\section{EMPIRICAL ANALYSIS}

\subsection{Variation among developers}

As seen in Fig. 1, there are some important differences in the frequency of emoticon usage between both Apache and Mozilla developers. However, we find that there are also Apache developers who have used emoticons in more than $10 \%$ of their comments.

Mozilla developers are usually more active on their issue tracker than Apache developers. The difference can be explained by the fact that Mozilla forces developers to use the issue tracker for code review. On the other hand, Apache gives more freedom to individual projects to handle modifications and code review. For the remaining of this section, we only keep the developers having written at least 100 comments. This leaves 727 Apache developers (76\%) and 462 (98\%) Mozilla developers, having written respectively $99.3 \%$ and $99.9 \%$ of all the comments. Even after filtering out the developers with less than 100 comments, there is still a statistically significant ( $p$-value < 0.001) difference between Apache and Mozilla developers

$\overline{{ }^{5} \text { https://en.wikipedia.org/wiki/List_of_emoticons }}$ 


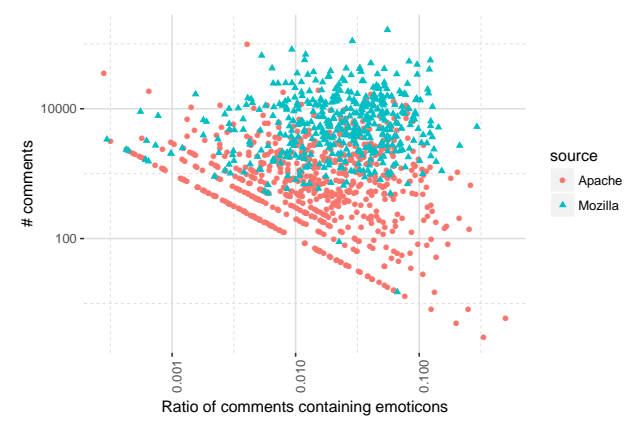

Figure 1: Number of comments and ratio of comments with at least one emoticon for each developer.

with a medium effect size (Cliff's $\delta=0.39$ ). After filtering out the developers who haven't used any emoticon, Mozilla developers still use emoticons significantly more often than Apache developers with a small effect size (Cliff's $\delta=0.27$ ).

Specifically for Mozilla, we manually extracted information about developers that were hired by the Mozilla Corporation in order to find out if we could identify demographic characteristics that can explain the differences of emoticon usage between developers. We didn't find any statistical differences between hired and volunteer developers. Moreover, for hired developers we also didn't find any statistically significant difference in terms of gender, position (managers vs. non manager, senior vs. non senior) or experience. We only observe a significant difference $(\mathrm{p}$-value $=0.002)$ between developers based in Europe or North America, and developers in Asia (Taiwan and Japan) or Oceania (Australia and New Zealand) with a medium size effect (Cliff's $\delta=-0.41$ ). As seen in Fig. 2, western developers are more likely to use emoticons than eastern developers.

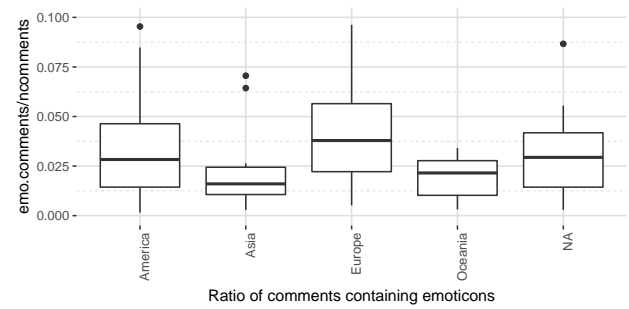

Figure 2: Ratios of comments with at least emoticons for hired Mozilla developers based on continents.

\subsection{Emotions expressed}

In accordance with the literature, the majority of emoticons are used to express joy. As seen in Table 2, besides joy, emoticons are mostly used to express sadness and surprise. We also looked at anger emoticons but these were used less than $1 \%$ of the time. Differences exist between the Apache and Mozilla communities. In Apache, more than $90 \%$ of the emoticons express joy. In Mozilla where emoticons are more frequently used than in Apache, sad
(15\%) and surprised (7\%) emoticons are also used more frequently than in Apache (7\% and 2\%).

Table 2: Percentage of emoticons used to express joy, sadness and surprise.

\begin{tabular}{l|rrrr} 
source & \% joy & \% sadness & \% surprise & \% other \\
\hline Apache & 90.13 & 7.27 & 2.3 & 0.34 \\
Mozilla & 78.29 & 14.93 & 6.73 & 0.05
\end{tabular}

There are also differences in term of emotions expressed between developers. 50.3\% of Apache developers, who have used emoticons, have only used them to express joy, while only $7.4 \%$ of the Mozilla developers have. $28.4 \%$ of the Mozilla developers have used more than $90 \%$ of emoticons to express joy and $73.5 \%$ of the Apache. On the other extreme, $6.1 \%$ of the Mozilla developers and 5.6\% Apache developers have used at least as many sad and surprised emoticons as joyful ones.

\subsection{Variation across the time of the week}

Table 3: Statistical difference (p-vlaue and Cliff's $\delta$ of MannWhitney $U$ test) between the ratio of issue comments with emoticons during the weekend and during the week.

\begin{tabular}{l|r|r|r|r} 
Source & Emoticons & Joy & Sadness & Surprised \\
\hline Mozilla & $0.17(0.04)$ & $0.08(-0.05)$ & $<0.001(0.15)$ & $<0.001(0.12)$ \\
Apache & $<0.001(0.22)$ & $<0.001(-0.19)$ & $<0.001(0.18)$ & $<0.001(0.11)$
\end{tabular}

Table 3 shows the p-value and effect sizes of a Mann-Whitney $\mathrm{U}$ test between the ratio of developers' comments with emoticons during the weekend and during weekdays. It is for Apache where the differences are more statistically significant and have stronger effect sizes. Emoticons are used more often in Apache projects during the weekend than during weekdays. Fig. 3 shows that this difference is observable in Apache because there are a number of developers with a relatively small percentage of comments made during weekends (5 to $20 \%$ ) but a large percentage of emoticons used during weekends ( $>25 \%$ ). On the other hand, weekends have a lower proportion of joyful emoticons and high proportions of sad and surprised emoticons. This difference is observed in Apache, and in Mozilla projects only for sad and surprised emoticons.

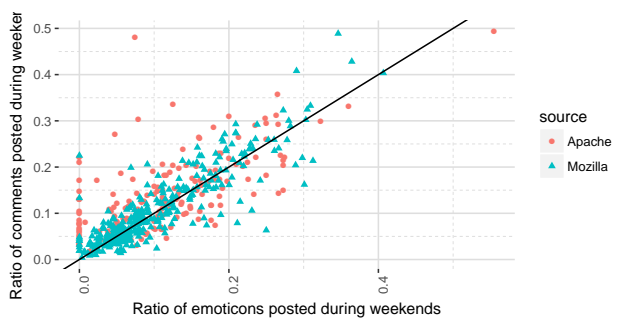

Figure 3: Developers' ratio of comments during weekends and ratio of emoticons during weekends. 


\section{THREATS TO VALIDITY}

We merged developers' identities using a very basic technique. Although we manually checked for false positives, there might be false negatives remaining. We only identified the most common ASCII emoticons and might have missed less common emoticons. Moreover, we manually classified emotions associated with emoticons and only associated one emotion to each emoticon. Differences of context (irony, sarcasm or different interpretation by the users) could imply a different meaning or emotion for some emoticons. Our study only includes open source projects from Apache and Mozilla. The results obtained are specific to those organization's culture and developer habits (external validity). Thus we cannot guarantee that our data set would be representative of the entire software industry or even open source industry.

\section{CONCLUSION AND FUTURE WORK}

In this paper, we conducted a preliminary investigation of the use of emoticons by software developers in Apache and Mozilla issue trackers. First, most Mozilla developers have used at least one emoticon, while more than $30 \%$ of the Apache developers never used any. There are also differences between individual developers and individual projects. We looked specifically at Mozilla's developers but couldn't find any statistical difference showing that professional developers might use emoticons differently than volunteers. Looking specifically at Mozilla's hired developers, we found only a difference between developers based in North America or Europe, and Asia or Oceania. The former using emoticons more frequently than the latter with a medium effect size. These differences between developers show that emoticons are most likely to act as a good proxy for sentiment analysis for developers frequently relying on emoticons.

In accordance to previous work, Mozilla and Apache developers mostly use emoticons to express joy. More than $90 \%$ of the emoticons used by Apache developers express joy and 78\% for Mozilla developers. Most other emoticons are used to indicate either sadness (7\% for Apache and 15\% for Mozilla) or surprise (2\% for Apache and $7 \%$ for Mozilla). The presence of a significant amount of sad and surprised emoticons show that it is potentially feasible to partly detect negative emotions such as frustration of developers without advanced sentiment analysis. We found a small difference in emoticon usage during the weekend and weekdays. During weekends, developers use more emoticons but these are less positives and include more sad or surprised emoticons.

As this paper is only a first step towards understanding how emoticons are used in software development, we will extend our research in different directions. First we want to study the context in which software developers are more likely to use emoticons. For this, we plan on conducting a survey and ask directly to software developers how they use emoticons. Second, we want to compare emoticon usage with the result of sentiment analysis tools. Finally we also intend find correlate emoticons usage with sentiment polarity, such as valence and arousal.

\section{ACKNOWLEDGMENTS}

The authors have been supported by Academy of Finland grant 298020 .

\section{REFERENCES}

[1] N. Novielli, F. Calefato, and F. Lanubile, "The challenges of sentiment detection in the social programmer ecosystem," in Proceedings of the 7th International Workshop on Social Software Engineering, ser. SSE 2015. New York, NY, USA: ACM, 2015, pp. 33-40. [Online]. Available: http: //doi.acm.org/10.1145/2804381.2804387

[2] R. Jongeling, S. Datta, and A. Serebrenik, "Choosing your weapons: On sentiment analysis tools for software engineering research," in Software maintenance and evolution (ICSME), 2015 IEEE international conference on. IEEE, 2015, pp. 531-535.

[3] M. R. Islam and M. F. Zibran, "Leveraging automated sentiment analysis in software engineering," in Proceedings of the 14th International Conference on Mining Software Repositories. IEEE Press, 2017, pp. 203-214.

[4] F. Calefato, F. Lanubile, F. Maiorano, and N. Novielli, "Sentiment polarity detection for software development," Empirical Software Engineering, vol. 23, no. 3, pp. 1352$1382,2018$.

[5] B. Lin, F. Zampetti, G. Bavota, M. Di Penta, M. Lanza, and R. Oliveto, "Sentiment analysis for software engineering: How far can we go?" in Proceedings of 40th International Conference on Software Engineering, 2018.

[6] B. Felbo, A. Mislove, A. Søgaard, I. Rahwan, and S. Lehmann, "Using millions of emoji occurrences to learn any-domain representations for detecting sentiment, emotion and sarcasm," arXiv preprint arXiv:1708.00524, 2017.

[7] N. Imtiaz, J. Middleton, P. Girouard, and E. Murphy-Hill, "Sentiment and politeness analysis tools on developer discussions are unreliable, but so are people," 2018.

[8] M. Boia, B. Faltings, C.-C. Musat, and P. Pu, "A:) is worth a thousand words: How people attach sentiment to emoticons and words in tweets," in Social computing (socialcom), 2013 international conference on. IEEE, 2013, pp. 345-350.

[9] J. Park, V. Barash, C. Fink, and M. Cha, "Emoticon style: Interpreting differences in emoticons across cultures." in ICWSM, 2013.

[10] L.-E. Marvin, "Spoof, spam, lurk, and lag: The aesthetics of text-based virtual realities," Journal of Computer-Mediated Communication, vol. 1, no. 2, p. JCMC122, 1995.

[11] L. Rezabek and J. Cochenour, "Visual cues in computer-mediated communication: Supplementing text with emoticons," Journal of Visual Literacy, vol. 18, no. 2, pp. 201-215, 1998.

[12] D. F. Witmer and S. L. Katzman, "On-line smiles: Does gender make a difference in the use of graphic accents?" Journal of Computer-mediated communication, vol. 2, no. 4, p. JCMC244, 1997.

[13] A. Hogenboom, D. Bal, F. Frasincar, M. Bal, F. de Jong, and U. Kaymak, "Exploiting emoticons in sentiment analysis," in Proceedings of the 28th Annual ACM Symposium on Applied Computing. ACM, 2013, pp. 703-710.

[14] S. Bahri, P. Bahri, and S. Lal, "A novel approach of sentiment classification using emoticons," Procedia Computer Science, vol. 132, pp. 669-678, 2018.

[15] P. K. Novak, J. Smailović, B. Sluban, and I. Mozetič, "Sentiment of emojis," PloS one, vol. 10, no. 12, p. e0144296, 2015.

[16] A. Hogenboom, D. Bal, F. Frasincar, M. Bal, F. De Jong, and U. Kaymak, "Exploiting emoticons in polarity classification of text." f. Web Eng., vol. 14, no. 1\&2, pp. 22-40, 2015.

[17] K.-L. Liu, W.-J. Li, and M. Guo, "Emoticon smoothed language models for twitter sentiment analysis." in Aaai, 2012.

[18] D. Davidov, O. Tsur, and A. Rappoport, "Enhanced sentiment learning using twitter hashtags and smileys," in Proceedings of the 23rd international conference on computational linguistics: posters. Association for Computational Linguistics, 2010, pp. 241-249.

[19] H. Wang and J. A. Castanon, "Sentiment expression via emoticons on social media," in Big Data (Big Data), 2015 IEEE International Conference on. IEEE, 2015, pp. 2404-2408.

[20] J. Read, "Using emoticons to reduce dependency in machine learning techniques for sentiment classification," in Proceedings of the ACL student research workshop. Association for Computational Linguistics, 2005, pp. 43-48.

[21] A. Wolf, "Emotional expression online: Gender differences in emoticon use," CyberPsychology \& Behavior, vol. 3, no. 5, pp. 827-833, 2000.

[22] H. Miller, J. Thebault-Spieker, S. Chang, I. Johnson, L. Terveen, and B. Hecht, "Blissfully happy" or "ready to fight": Varying interpretations of emoji," Proceedings of ICWSM, vol. 2016, 2016.

[23] S. Dueñas, V. Cosentino, G. Robles, and J. M. Gonzalez-Barahona, "Perceval: Software project data at your will," in Proceedings of the 40th International Conference on Software Engineering: Companion Proceeedings, ser. ICSE '18. New York, NY, USA: ACM, 2018, pp. 1-4. [Online]. Available: http://doi.acm.org/10.1145/3183440.3183475

[24] M. V. Mäntylä, F. Calefato, and M. Claes, "Natural language or not (nlon)a package for software engineering text analysis pipeline," arXiv preprint arXiv:1803.07292, 2018.

[25] The emotions. University Press of America, 1991. 